\title{
Snapin Mediates Insulin Secretory Granule Docking, but not trans-SNARE Complex Formation
}

Running title: Role of snapin in insulin secretion

Sangeeta Somanath ${ }^{1}$, Christopher J. Partridge ${ }^{2}$, Catriona Marshall ${ }^{1}$, Tony Rowe ${ }^{3}$ and Mark D. Turner ${ }^{4}$

${ }^{1}$ Blizard Institute, Barts and The London School of Medicine and Dentistry, Queen Mary University of London, London, E1 2AT, UK. ${ }^{2}$ Diabetes Research Laboratories, Oxford Centre for Diabetes, Endocrinology and Churchill Hospital, University of Oxford, Oxford, OX3 7LJ, UK. ${ }^{3}$ CSL Limited, 45 Poplar Road, Parkville, Victoria 3052, Australia. ${ }^{4}$ Interdisciplinary Biomedical Research Centre, School of Science and Technology, Nottingham Trent University, Nottingham, NG11 8NS, UK

Correspondence to:

Dr. Mark D. Turner,

Interdisciplinary Biomedical Research Centre,

School of Science and Technology,

Nottingham Trent University,

Nottingham,

NG11 8NS,

United Kingdom.

e-mail: mark.turner@ntu.ac.uk

Tel: +44 (0)115 848-3113 


\begin{abstract}
Secretory granule exocytosis is a tightly regulated process requiring granule targeting, tethering, priming, and membrane fusion. At the heart of this process is the SNARE complex, which drives fusion through a coiled-coil zippering effect mediated by the granule v-SNARE protein, VAMP2, and the plasma membrane t-SNAREs, SNAP-25 and syntaxin-1A. Here we demonstrate that in pancreatic $\beta$-cells the SNAP-25 accessory protein, snapin, $C$-terminal $\mathrm{H} 2$ domain binds SNAP-25 through its $\mathrm{N}$ terminal Sn-1 domain. Interestingly whilst snapin binds SNAP-25, there is only modest binding of this complex with syntaxin-1A under resting conditions. Instead synataxin-1A appears to be recruited in response to secretory stimulation. These results indicate that snapin plays a role in tethering insulin granules to the plasma membrane through coiled coil interaction of snapin with SNAP-25, with full granule fusion competency only resulting after subsequent syntaxin-1A recruitment triggered by secretory stimulation.
\end{abstract}

Key Words: exocytosis, SNAP-25, secretory granule tethering 


\section{Introduction}

Snapin is a $15 \mathrm{KDa}$ protein that has been proposed to fulfill multiple signal transduction and endomembrane trafficking/fusion functions. However, central to most roles of snapin is its association with the target soluble $N$-ethyl maleimide sensitive fusion protein attachment receptor (t-SNARE), SNAP-25 [1]. In addition, in neurons snapin has also been reported to play a key role in the recruitment of the $\mathrm{Ca}^{2+}$ sensor, synaptotagmin-1, to the SNARE complex through enhanced synaptotagmin-1SNAP-25 binding [2], resulting in stabilization of the release-ready pool of secretory vesicles. Moreover, snapin plays a dual role in neurons, both stabilizing secretory vesicle priming at the plasma membrane and also synchronizing secretory vesicle fusion [3]. The role of snapin in the regulation of endocrine cell secretion is however less clear, as synaptotagmin-1 is generally expressed at much lower levels in these cells [4]. The current study examines the role of snapin in the regulation of hormone secretion from one such endocrine cell, the insulin secreting pancreatic $\beta$-cell, and addresses the nature of snapin interactions with members of the SNARE complex in secretory granule docking and exocytosis. 


\section{Results and Discussion}

\section{Snapin is primarily associated with insulin-containing secretory granules}

Snapin was initially reported as being a transmembrane protein regulating synaptic transmission [1,5]. However, subsequent studies have shown snapin to be widely expressed, and to function as a more general component of vesicle trafficking pathways, including regulating SNAP-25 and SNAP-23 dependent transport steps in multiple cell types. In 3T3-L1 adipocytes, biochemical and immunofluorescence microscopy analysis showed it to reside within both diffuse cytosolic and perinuclear membrane compartments [6]. Snapin has also been shown to be expressed in other endocrine cells, including rat and mouse pancreatic insulinoma cells, as well as primary mouse and human islets, although not in exocrine tissue. Furthermore it was found to predominantly co-localise with insulin in mouse islets [7]. Consistent with these observations, we found that snapin exhibited some diffuse cytosolic staining as well as being clustered into punctate structures within the cell body of rat INS-1 cells. Furthermore, the punctate staining pattern was similar to that seen for insulincontaining secretory granules (Fig. 1). Indeed when the two fields were then superimposed, analysis confirmed that there was significant co-fluorescence between the punctate insulin and snapin signals, indicative of co-localization of snapin and insulin to $\beta$-cell secretory granules.

\section{Insulin secretion is dependent upon snapin H2 domain interaction with SNAP-25}

Even though snapin does not appear to primarily localize to sites of exocytosis in pancreatic $\beta$-cells, snapin interaction with SNAP-25 is nevertheless well documented in a wide range of cell types. In order to determine the role of snapin in secretion, we investigated the effect of transfecting a range of His-tagged snapin domain deletion 
constructs into INS-1 cells. Using the Coils programme [8], snapin has been reported to contain an $\mathrm{N}$-terminal hydrophobic domain and two helical domains (H1; amino acids 37-65, and H2; amino acids 81-126) [6]. We transfected INS-1 cells with pcDNA4 vector containing either full length wild type snapin[1-136], snapin[1-79] comprising the $\mathrm{N}$-terminal hydrophobic region and H1, snapin[36-136] comprising both predicted helical domains, snapin[79-136] comprising H2 only, or control vector containing His-tagged $\beta$-galactosidase (Fig 2A). Western blot analysis using anti-V5 antibody indicated that all constructs were transfected efficiently, and with similar expression levels (Fig. 2B).

Transfected and control cells were next incubated with secretagogue cocktail, and insulin secretion determined by ELISA analysis of media. We found that transfection of full length wild type snapin resulted in a moderate increase in secretagoguestimulated secretion, whereas transfection of truncated mutants led to inhibition in insulin secretion (Fig. 2C). Importantly, snapin[1-79] only modestly inhibited secretion, whereas expression of snapin[36-136] and snapin[79-136] resulted in $>50 \%$ inhibition. As the latter two constructs both contain the $C$-terminal predicted $\mathrm{H} 2$ helical domain, this data indicates that the primary SNAP-25 binding site of snapin lies within the H2 domain.

\section{Snapin binding with SNAP-25 precedes granule exocytosis}

In keeping with previously reports of its association with SNAP-25 in neuronal cells [1] and SNAP-23 in certain non-neuronal cells [6], snapin has also previously been shown to interact with SNAP-25 in pancreatic $\beta$-cells [7]. Interestingly, the study showed that snapin knock-down using RNAi inhibited insulin secretion, thereby 
demonstrating an essential role for snapin in secretagogue-stimulated insulin secretion. Although the authors went on to examine the relationship of snapin phosphorylation with its association to SNAP-25 and the secretory granule protein, VAMP2, there are to our knowledge currently no reports documenting the role of snapin in $\beta$-cell SNAP25/syntaxin 1A t-SNARE interactions.

In order to determine the role of snapin interaction with SNAP-25, and its implications for SNARE complex dynamics, we immunoprecipitated SNAP-25 and then immunoblotted with affinity purified antibody raised against snapin, and found that there was an association between the two proteins in both unstimulated and secretagogue-stimulated INS-1 cells (Fig. 3A). Similarly, when we immunoprecipitated snapin and then immunoblotted with antibody raised against SNAP-25, we also found that there was a strong interaction between the two proteins in both unstimulated and secretagogue-stimulated cells (Fig. 3B). Importantly, we have previously shown that exocytosis in pancreatic $\beta$-cells is accompanied by partial proteolysis of SNAP-25 [9]. Interestingly, while we again observed SNAP-25 cleavage in immunoblots of INS-1 cells incubated with secretagogue cocktail (Fig. 3C), only full length, and not partially cleaved SNAP-25, was found to coimmunoprecipitate with snapin (Fig. 3B). The absence of an interaction between snapin and the partially proteolysed SNAP-25 protein therefore indicates that snapin binds to SNAP-25 via its $N$-terminal Sn1 domain, as it is within this region of SNAP25 that we have previously found SNAP-25 to be proteolytically cleaved by calpain-10 during exocytosis [9]. As such the larger clipped fragment of SNAP-25, lacking its $N$ terminus, is no longer observed on Western blots of samples immunoprecipitated with snapin following secretagogue-stimulated partial proteolysis. 
In contrast to SNAP-25 binding, the integral membrane t-SNARE, syntaxin-1, only weakly associates with snapin under resting conditions. However when cells are incubated with secretagogue cocktail there is a significant increase in association between these two molecules (Fig. 3D). These observations have important implications, as this indicates that when $\beta$-cell secretory granules dock with the plasma membrane, granules are bound to SNAP-25 without the presence of significant additional interaction between SNAP-25 and syntaxin 1. It is therefore likely that the interaction between snapin and SNAP-25 serves, at least in part, to tether granules to the plasma membrane. Contrary to mainstream conventional thinking, this would also suggest that docked insulin granules do not extensively form trans-SNARE complexes prior to exocytosis. This hypothesis is not without precedence however, as snapin is able to bind SNAP-25 in both free and SNARE complex aggregate conformations [10], potentially enabling it to function in either mode of action dependent upon cell type and intracellular environment. Our current data is also consistent with snapin interactions with synaptotagmin in C. elegans, which unlike previous findings in neurons $[1,2]$ have shown synaptotagmin interaction to lie downstream of snapinSNAP-25 mediated vesicle tethering [11]

The formation of fully zippered SNARE complex is known to be sufficient to catalyse bilayer fusion [12]. Therefore, it is crucial that cells strictly regulate spatial and temporal complex formation. Knowledge of the regulatory molecular machinery mediating SNARE assembly continues to grow, with munc18a having been shown to block SNARE complex assembly when bound to N-peptide of the $N$-terminal domain of syntaxin-1A [13]. This indicates a physiological role for munc18 to prevent 
premature full SNARE complex assembly. Interestingly, munc18c also inhibits GLUT4 vesicle fusion in adipocytes [14], with tyrosine 521 phosphorylation dissociating the syntaxin/munc18c complex and attenuating inhibition of vesicle fusion [15]. Importantly, munc18c phosphorylation has also been suggested to be a trigger for insulin granule fusion in $\beta$-cells [16]. This data is consistent with a model in which granule docking is mediated by snapin/SNAP-25 binding, with subsequent full SNARE complex formation only occurring later when secretory stimulation triggers munc-18 phosphorylation and release of syntaxin from munc/syntaxin complex, and thus facilitating full SNARE complex formation in the process.

Snapin is known to bind to the t-SNARE, SNAP-25, yet immunofluorescence shows that the primary localization of snapin in $\beta$-cells is with secretory granules. As it has previously been documented that secretory granules track along cytoskeletal microtubules in $\beta$-cells $[17,18]$, linear granule movement is likely to continue back and forth until snapin binds to SNAP-25 at the plasma membrane, thereby clamping granules in a morphologically docked plasma membrane granule pool through $\mathrm{H} 2$ domain coiled-coil interactions of snapin with the $N$-terminal Sn1 domain of SNAP25. Snapin/SNAP-25 tethered granules also likely represent the docked but non-readily releasable pool that constitutes $\sim 95 \%$ of secretory granules located at the $\beta$-cell plasma membrane [19]. Subsequent secretagogue-stimulated munc18c phosphorylation would then release syntaxin-1A for full SNARE complex formation, thereby imparting docked insulin granules with fusion competency. 


\section{Methods}

\section{Materials}

Snapin constructs were subcloned into pcDNA4 vector (Invitrogen, Carlsbad, CA), as described previously [6]. Antibodies used were as follows: mouse anti-Xpress (Invitrogen, Carlsbad, CA), mouse anti SNAP-25 (BD Biosciences, San Diego, CA), goat anti SNAP-25 (Santa Cruz Biotechnology, Santa Cruz, CA), rabbit anti-snapin (purified as described in [6]), goat anti-rabbit-HRP (Bio-Rad, Hercules, CA), rabbit anti-goat-HRP conjugate (Dako, Ely, UK), goat anti-mouse-HRP conjugate (Dako, Ely, UK), peroxidase-conjugated anti-rabbit antisera (Dako, Ely, UK), swine antirabbit FITC (Dako, Ely, UK), and Guinea pig anti- insulin (Linco, St. Charles, MO). Insulin ELISA kit was from Mercodia (Uppsala, Sweden). Unless stated otherwise all other reagents were from Sigma (St. Louis, MO).

\section{Snapin expression}

INS-1 cells were grown until 70-80\% confluence, then electroporated using the T-020 programme of an Amaxa electroporator. The process was repeated for tagged full length wild-type snapin, truncated snapin constructs, or control His-tagged $\beta$ galactosidase vector. After 48h, cells were lysed in RIPA buffer, and cell debris removed. Cell homogenates were boiled in Laemmli sample buffer (after normalizing samples for cellular protein content) and separated on 10\% SDS-PAGE gels. Protein was transferred onto PVDF membrane using a Hoefer TE 70 semi-dry transfer unit (Amersham Pharmacia, Little Chalfont, UK) and the presence of tagged snapin emanating from the pcDNA4 vector detected by immunoblotting with anti-Xpress antibody (1:1500). Antibody binding was detected using HRP-conjugated secondary antibody (1:1000) and visualized using ECL plus chemiluminescent detection and 
Hyperfilm ECL (Amersham Pharmacia, Little Chalfont, UK). Band intensity was quantified using an IS-1000 Digital Imaging System (Alpha Innotech Corporation, San Leandro, CA).

\section{Co-immunoprecipitation of SNAP-25 and snapin}

INS-1 cells were grown to $80 \%$ confluence in poly-D-lysine coated 6-well plates. Either $15 \mu 1$ of anti-SNAP-25 (C-terminal) antibody was mixed with $100 \mu$ Protein G Sepharose beads, or $15 \mu 1$ of anti-snapin antibody was mixed with $100 \mu 1$ Protein A Sepharose beads. Samples were rotated for $2 \mathrm{~h}$ at $4^{0} \mathrm{C}$. Cells were lysed in $1 \mathrm{ml}$ of NP40 buffer (150mM NaCl, 50mM Tris Base, 1\% Triton X-100, plus Complete protease cocktail) and the supernatant mixed with antibody-bead mix and rotated overnight at $4^{0} \mathrm{C}$. Samples were then washed with IP buffer and proteins separated on $10 \%$ SDSPAGE gel and Western blotting performed as detailed previously.

\section{Snapin immunofluorescence}

INS-1 cells were cultured on permanox or poly-L-lysine coated 8-well Lab-Tek chamber slides (Nalgene-Nunc, Rochester, NY). Following incubation, cells were fixed in $4 \%$ paraformaldehyde in phosphate buffer, permeabilized with $0.1 \%$ Triton X-100 in PBS containing 5\% swine serum at $4^{\circ} \mathrm{C}$ and incubated overnight with antisnapin antibody (1:30 dilution) or anti-insulin (1:100 dilution). Snapin was visualized by incubation for 1 hour with swine anti-rabbit FITC (1:30), or insulin with texas redconjugated goat anti-guinea pig (1:50), and washed in PBS. Following removal of the wells, the slides were mounted in Vectashield (Vector Labs, Burlingame, CA) and fluorescence was viewed under a Leica TCS-NT laser scanning confocal microscope. 


\section{Insulin secretion}

Cells were seeded onto poly-D-lysine coated 6-well plates and allowed to grow to near confluency $\left(2 \times 10^{6}\right.$ cells/well). Cells were then washed with Krebs-Ringer solution $\left(125 \mathrm{mM} \mathrm{NaCl}, 1.2 \mathrm{mM} \mathrm{KH} \mathrm{PO}_{4}, 5 \mathrm{mM} \mathrm{KCl}, 2 \mathrm{mM} \mathrm{MgSO}\right.$, $1.67 \mathrm{mM}$ glucose, 0.1\% BSA, 25 mM HEPES; pH 7.4) and incubated for $3 \mathrm{~h}$ in Krebs-Ringer solution +/- insulin secretagogue cocktail (10 mM glucose, $1 \mu \mathrm{M}$ phorbol 12 myristate 13-acetate, $1 \mathrm{mM}$ isobutyl-methylxanthine, $1 \mathrm{mM}$ tolbutamide, $10 \mathrm{mM}$ leucine, $10 \mathrm{mM}$ glutamine, $1 \mathrm{mM} \mathrm{CaCl}_{2}$ ). Supernatant was collected, spun down to remove cell debris, Complete protease cocktail (Roche, Basel, Switzerland) added and insulin content determined following standard ELISA protocol (Mercodia, Uppsala, Sweden). Cellular protein content was assayed from lysed unstimulated cells as per BCA kit protocol (Pierce Biotechnology Inc., Rockford, IL) and used to normalize secretion data.

\section{Statistical analysis}

Analysis between 2 groups was by independent samples t-test. Comparison between more than 2 groups was by one way ANOVA, followed by Dunnett t post-hoc test. Linear correlation was determined using Spearman's rho correlation coefficient. All experimental data was gathered from a series of independent experiments $(n=7)$. 


\section{Acknowledgements}

This research was supported by Barts and The London Charitable Foundation (RAC RAB/06/PHD/12). The authors declare that they have no conflict of interest.

\section{Author Contributions}

Sangeeta Somanath: Acquisition of data, manuscript construction and approval.

Christopher J. Partridge: Acquisition of data, manuscript construction and approval.

Catriona Marshall: Acquisition of data, manuscript construction and approval.

Tony Rowe: Experimental design, manuscript construction and approval.

Mark D. Turner: Experimental design, data analysis and interpretation, manuscript construction and approval. 


\section{References}

[1] J.M. Ilardi, S. Mochida, Z.H. Sheng. Snapin: a SNARE-associated protein implicated in synaptic transmission. Nat. Neurosci. 2 (1999) 119-124.

[2] J.H. Tian, Z.X. Wu, M. Unzicker, L. Lu, Q. Cai, C. Li, C. Schirra, U. Matti, D. Stevens, C. Deng, J. Rettig, Z.H. Sheng. The role of snapin in neurosecretion: snapin knock-out mice exhibit impaired calcium-dependent exocytosis of large dense-core vesicles in chromaffin cells. J. Neurosci. 5 (2005) 10546-10555.

[3] P.Y. Pan, J.H. Tian, Z.H. Sheng. Snapin facilitates the synchronization of synaptic vesicle fusion. Neuron 61 (2009) 412-424.

[4] E. Aganna, J.M. Burrin, G.A. Hitman, M.D. Turner. Involvement of calpain and synaptotagmin $\mathrm{Ca}^{2+}$ sensors in hormone secretion from excitable endocrine cells. J. Endocrinol. 191 (2006) R1-7.

[5] S. Hilfiker, P. Greengard, G.J. Augustine. Coupling calcium to SNARE-mediated synaptic vesicle fusion. Nat. Neurosci. 2 (1999) 104-106.

[6] P. Buxton, X.M. Zhang, B. Walsh, A. Sriratana, I. Schenberg, E. Manickam, T. Rowe. Identification and characterization of Snapin as a ubiquitously expressed SNARE-binding protein that interacts with SNAP23 in non-neuronal cells. Biochem. J. 375 (2003) 433-440. 
[7] W.J. Song, M. Seshadri, U. Ashraf, T. Mdluli, P. Mondal, M. Keil, M. Azevedo, L.S. Kirschner, C.A. Stratakis, M.A. Hussain. Snapin mediates incretin action and augments glucose-dependent insulin secretion. Cell Metab. 13 (2011) 308-319.

[8] A. Lupas, M. Van Dyke, J. Stock. Predicting coiled coils from protein sequences. Science 252 (1991) 1162-1164.

[9] C. Marshall, G.A. Hitman, C.J. Partridge, A. Clark, H. Ma, T.R. Shearer, M.D. Turner. Evidence that an isoform of calpain-10 is a regulator of exocytosis in pancreatic $\beta$-cells. Mol. Endocrinol. 19 (2005) 213-224.

[10] A. Navarro, J.A. Encinar, B. López-Méndez, D. Aguado-Llera, J. Prieto, J. Gómez, L.A. Martínez-Cruz, O. Millet, J.M. González-Ros, G. Fernández-Ballester, J.L. Neira, A. Ferrer-Montiel. Mutation of Ser-50 and Cys-66 in Snapin modulates protein structure and stability. Biochemistry 51 (2012) 3470-3484.

[11] S.C. Yu, S.M. Klosterman, A.A. Martin, E.O. Gracheva, J.E. Richmond. Differential roles for snapin and synaptotagmin in the synaptic vesicle cycle. PLoS One 8 (2013) e57842.

[12] T. Weber, B.V. Zemelman, J.A. McNew, B. Westermann, M. Gmachl, F. Parlati, T.H. Sollner, J.E. Rothman. SNAREpins: minimal machinery for membrane fusion. Cell 92 (1998) 759-772. 
[13] P. Burkhardt, D.A. Hattendorf, W.I. Weis, D. Fasshauer. Munc18a controls SNARE assembly through its interaction with the syntaxin N-peptide. EMBO J. 27 (2008) 923-933.

[14] D.C. Thurmond, B.P. Ceresa, S. Okada, J.S. Elmendorf, K. Coker, J.E. Pessin. Regulation of insulin-stimulated GLUT4 translocation by Munc18c in 3T3L1 adipocytes. J. Biol. Chem. 273 (1998) 33876-33883.

[15] V. Aran, N.J. Bryant, G.W. Gould. Tyrosine phosphorylation of Munc18c on residue 521 abrogates binding to syntaxin 4. BMC Biochem. 12 (2011) 19.

[16] E. Oh, D.C. Thurmond. The stimulus-induced tyrosine phosphorylation of Munc18c facilitates vesicle exocytosis. J. Biol. Chem. 281 (2006) 17624-17634.

[17] A. Varadi, E.K. Ainscow, V.J. Allan, G.A. Rutter. Involvement of conventional kinesin in glucose-stimulated secretory granule movements and exocytosis in clonal pancreatic beta-cells. J. Cell Sci. 115 (2002) 4177-4189.

[18] A. Varadi, T. Tsuboi, L.I. Johnson-Cadwell, V.J. Allan, G.A. Rutter. Kinesin I and cytoplasmic dynein orchestrate glucose-stimulated insulin-containing vesicle movements in clonal MIN6 beta-cells. Biochem. Biophys. Res. Commun. 311 (2003) 272-282.

[19] T.K. Bratanova-Tochkova, H. Cheng, S. Daniel, S. Gunawardana, Y.J. Liu, J. Mulvaney-Musa, T. Schermerhorn, S.G. Straub, H. Yajima, G.W. Sharp. Triggering 
and augmentation mechanisms, granule pools, and biphasic insulin secretion. Diabetes 51 Suppl. 1 (2002) S83-90. 


\section{Figure Legends}

Figure 1: Snapin associates with insulin-containing secretory granules. Snapin was visualized in INS-1 cells using anti-snapin antibody in conjunction with swine antirabbit FITC, whereas insulin was visualized using polyclonal guinea pig anti-insulin and texas red-conjugated goat anti-guinea pig. Slides were mounted and fluorescence viewed under a laser scanning confocal microscope equipped with a $488 \mathrm{~nm}$ laser excitation filter for FITC. Scale bar, $2 \mu \mathrm{m}$.

Figure 2: Effect of snapin constructs upon insulin secretion. a) Domain map of snapin, indicating the positions of the N-terminal hydrophobic domain (HD; amino acids 1-20) and two predicted helical regions (H1 and H2; amino acids 37-65 and 81126, respectively). The truncated mutants were constructed by deleting specific domains of snapin as indicated. Cells were transfected with pcDNA4 vector containing $\beta$-galactosidase as a negative control. All vectors were His-tagged. b) INS-1 cells were transfected with tagged full length wild-type snapin, truncated snapin constructs, or $\beta$-galactosidase vector as control. After $48 \mathrm{~h}$, cells were lysed in RIPA buffer, cell debris removed, and protein then separated on 10\% SDS-PAGE and electrotransferred to PVDF. After blocking, PVDF membrane was immunoblotted with anti-Xpress antibody to detect His-tag. c) INS-1 cells were transfected with wild-type snapin, truncated snapin constructs, or empty vector as mock control. After 48h, cells were incubated for $3 \mathrm{~h}+/$-secretagogue cocktail. Supernatants were collected and insulin secretion quantified by ELISA, and normalized to cellular protein content. Statistical significance to mock control is indicated as follows: ${ }^{*} \mathrm{p}<0.05$, $\left.{ }^{* *} \mathrm{p}<0.01\right)$. 
Figure 3: Snapin immunoprecipitation with SNAP-25. a) INS-1 cells were cultured in RPMI-1640 media, then lysed in RIPA buffer, cell debris removed, and protein then separated on 10\% SDS-PAGE and electrotransferred to PVDF. After blocking, PVDF membrane was immunoblotted with antibody raised against $C$-terminal domain of SNAP-25, and antibody binding detected using ECL chemiluminescence. b, c), and d) INS-1 cells were lysed in NP-40 buffer, then protein immunoprecipitated using Protein A (snapin) or Protein G (SNAP-25 or syntaxin 1) beads coupled to antibody raised against either snapin, SNAP-25, or syntaxin 1. Samples were then washed with IP buffer and proteins separated on 10\% SDS-PAGE gel and electrotransferred to PVDF. After blocking, PVDF membrane was immunoblotted with antibody raised against either SNAP-25 or snapin. 

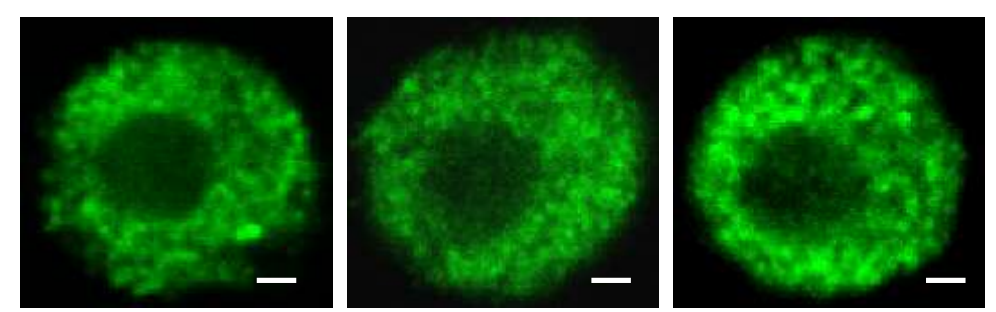

Snapin
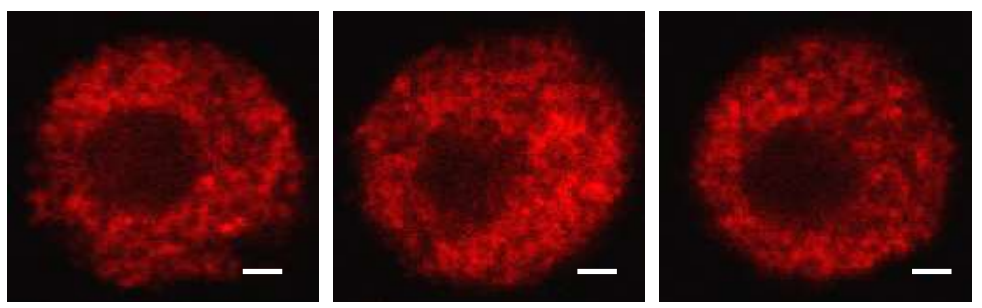

Insulin
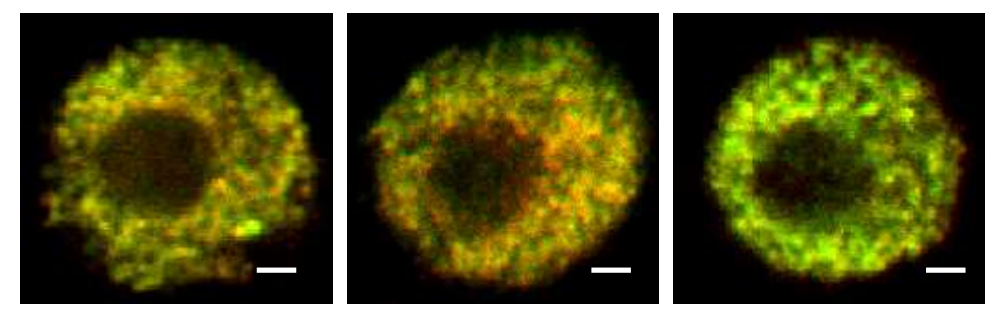

Merge

Figure 1: Somanath et al, 2016 


\section{Snapin Vectors}

A)

Wild type (1-136 aa)
Snapin (1-79 aa)
Snapin (36-136 aa)
Snapin (79-136 aa)

B)

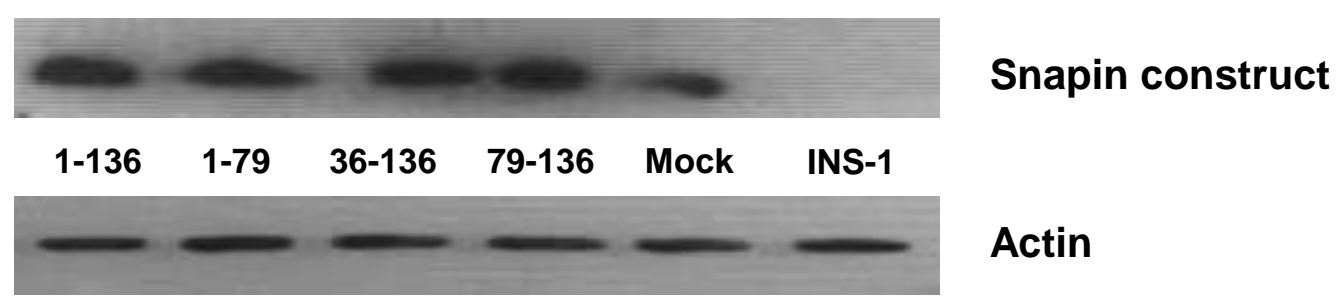

C)

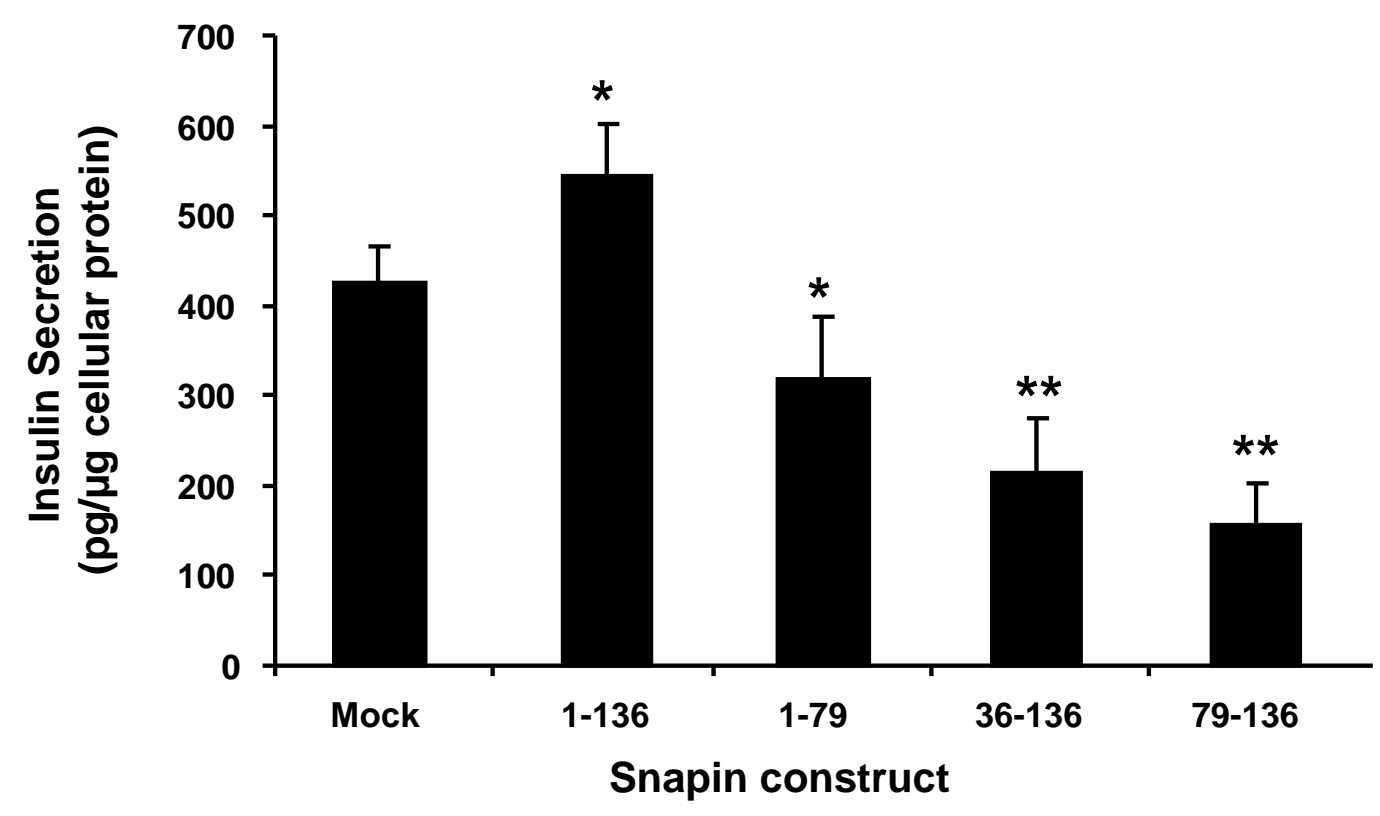

Figure 2: Somanath et al, 2016 
AP SNAP-25
WB Snapin

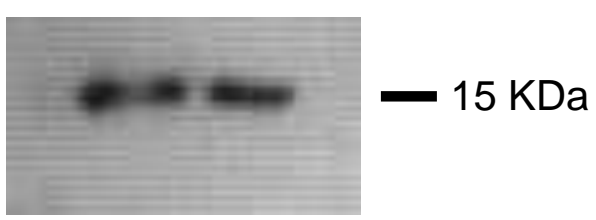

Unstim Stim

C) WB SNAP-25

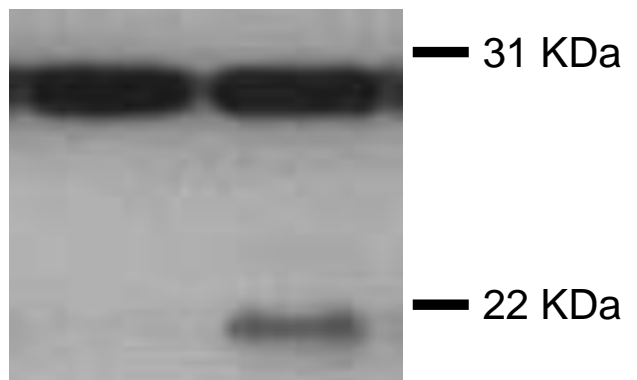

Unstim

$$
\text { Stim }
$$

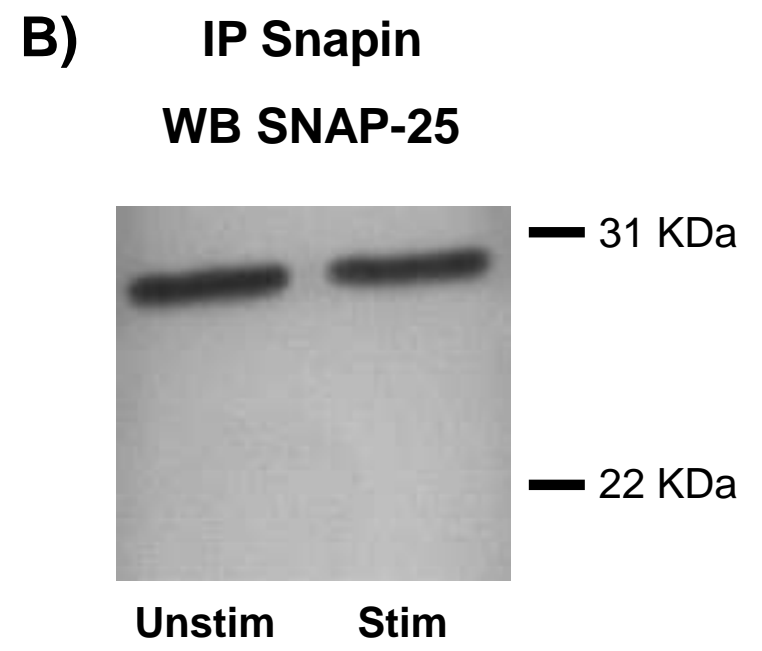

D) IP Syntaxin-1

WB Snapin

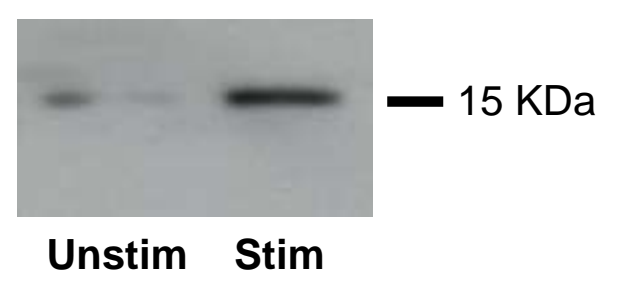

